\title{
Primary tooth abscess caused by Mycobacterium bovis in an immunocompetent child
}

\author{
Chrysoula Maragou • Nadia Theologie-Lygidakis • \\ Panayotis Ioannidis • Antonia Stenou • \\ Sophia Kanavaki $\cdot$ Ioannis Iatrou $\cdot$ Maria N. Tsolia
}

Received: 12 November 2009/Accepted: 3 February 2010/Published online: 4 May 2010

(C) The Author(s) 2010. This article is published with open access at Springerlink.com

\begin{abstract}
Bovine tuberculosis is a zoonotic disease, and although its incidence has dramatically decreased in developed countries where effective control measures are applied, it still remains a potential health hazard in the developing world. Tuberculosis of the oral cavity is extremely rare and is usually secondary to pulmonary involvement. We present the unusual case of an immunocompetent 6-year-old child residing in an urban area with primary oral tuberculosis due to Mycobacterium bovis, which was confirmed by the application of a molecular genetic approach. M. bovis belongs to Mycobacterium tuberculosis complex which comprises species with close genetic relationship, and for this reason, the use of new molecular techniques is a useful tool for the differentiation at species level of the closely related members of this complex.
\end{abstract}

\footnotetext{
C. Maragou $(\bowtie) \cdot$ A. Stenou

First Department of Pediatrics, "P. and A. Kyriakou"

Children's Hospital,

11527 Athens, Greece

e-mail: chrysa_mar@hotmail.com

N. Theologie-Lygidakis $\cdot$ I. Iatrou

Department of Oral and Maxillofacial Surgery (OMFS),

School of Dentistry, Athens University,

Athens, Greece

P. Ioannidis $\cdot$ S. Kanavaki

Microbiology Laboratory, Sotiria Chest Hospital,

Athens, Greece

\section{N. Tsolia}

Second Department of Pediatrics, P. and A. Kyriakou Children's Hospital, Athens University School of Medicine,

Athens, Greece
}

Keywords M. bovis $\cdot$ Dental abscess $\cdot$ Zoonoses . DNA-STRIP technology

\section{Introduction}

Both primary and secondary types of tuberculosis can cause lesions in the oral cavity [5]. In secondary tuberculosis, lesions of the oral cavity may accompany lesions in the pharynx, lungs, lymph nodes, or skin [5]. When oral lesions of tuberculosis are the sole manifestations of the disease, the clinician may face a diagnostic challenge. During the past decades, primary bovine tuberculosis of the oral cavity became extremely rare and occurs mainly through consumption of raw milk from infected cattle in developing countries [2]. The purpose of this report is to present an unusual case of primary oral tuberculosis due to Mycobacterium bovis in a 6-year-old boy residing in an urban area.

\section{Case report}

A 6-year-old, previously healthy boy, presented to the Pediatric department because of a strongly positive tuberculin skin test $(34 \mathrm{~mm})$ and mild chronic swelling with a skin fistula in the left submandibular area, which developed 3 months prior to admission. He was initially treated with oral and then intravenous broad spectrum antibiotics for 3 weeks with the presumptive diagnosis of cervical adenitis with no response at another hospital. After evaluation by an oral and maxillofacial surgeon, the diagnosis of a submandibular abscess originating from the decayed primary second lower left molar was made (Fig. 1a). The patient underwent incision and drainage of the abscess extraorally and extrac- 

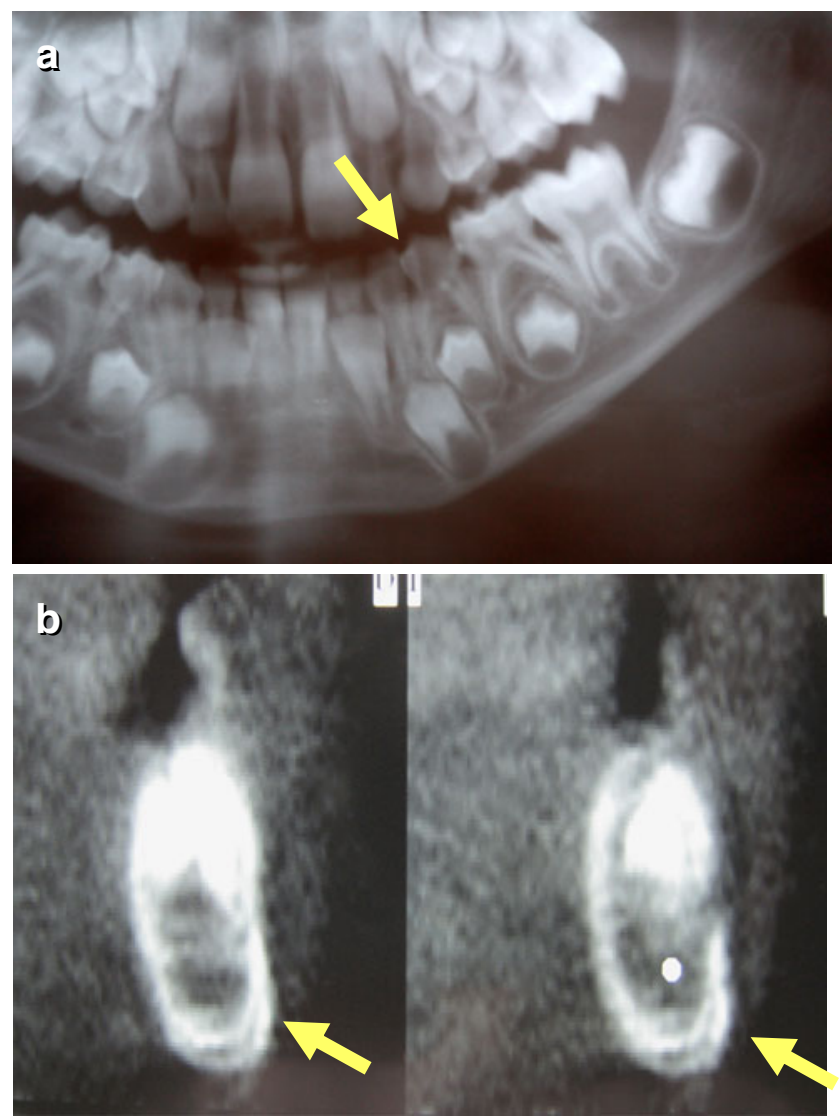

Fig. 1 Radiological imaging. a Panoramic x-ray showing the decayed primary second lower left molar and (b) computed tomography showing chronic osteomyelitis with osteoblastic subperiosteal activity and thickening around the lower mandibular border

tion of the involved tooth. Postoperatively, the symptoms improved, and he was discharged. Routine bacterial cultures were negative. The following 2 months, he had persistent moderate swelling, and a fistula developed at the incision site.

The patient had no history of contact with a case of active tuberculosis, and investigation of family contacts was negative. He lived in an urban area and had no contact with cattle or history of consumption of unpasteurized dairy products. The child was born in Greece and never travelled abroad. Both grandparents and parents were born in the former Soviet Union and had immigrated to Greece 20 years ago. The patient was not immunized with Bacillus CalmetteGuérin vaccine.

Physical examination on admission revealed a wellnourished boy in good clinical condition. A left submandibular firm, erythematous swelling measuring $3 \times 3 \mathrm{~cm}$, was palpated with a draining sinus tract. Laboratory studies were unremarkable. Blood tests revealed a white blood cell count of $15,200 / \mathrm{mm}^{3}$ with $74 \%$ polymorphonuclears, $18 \%$ lymphocytes, and $8 \%$ monocytes; erythrocyte sedimentation rate was $50 \mathrm{~mm} / \mathrm{h}$, and C-reactive protein was $77 \mathrm{mg} / \mathrm{L}$.
Measurement of serum immunoglobulins, determination of lymphocyte subpopulations by flow cytometry, and nitroblue tetrazolium test were performed and were normal. Serologic testing ruled out human immunodeficiency virus (HIV) 1 and 2 (enzyme-linked immunosorbent assay method), toxoplasmosis, cytomegalovirus, Epstein-Barr virus, and Bartonella henselae infection. Furthermore, chest radiography and abdominal ultrasound were normal, while culture of gastric aspirate was negative. QuantiFERON-TB Gold In-tube (QFT-IT) test was positive. A computed tomography of the region revealed the presence of chronic osteomyelitis of the mandibular body adjacent to the extraction site with osteoblastic subperiosteal activity and thickening around the lower mandibular border (Fig. 1b).

Antituberculous treatment was initiated with isoniazid, rifampicin, and pyrazinamide (PZA), and the patient underwent surgical excision of the submandibular mass and the draining sinus. The histopathological examination revealed no lymphoid but only fatty and fibrous tissue with granulomatous caseating infection. Acid fast bacilli were visualized by Ziehl-Neelsen stain. Two months later, mycobacterial growth was evident on Lowenstein-Jensen medium. The culture isolate was identified as M. bovis subsp. bovis by means of the GenoType Mycobacterium tuberculosis complex (MTBC) assay (Hain Lifescience, Germany). This assay is based on the DNA-STRIP technology and permits the genetic differentiation of species belonging to the Mycobacterium complex on the basis of gyrase B polymorphisms [8]. Our finding was subsequently verified in the central laboratories of the kit manufacturer. Susceptibility test showed resistance to PZA, as expected. Based on these results, PZA was discontinued, and ethambutol was added to the therapeutic regimen. By the end of a 6-month therapy, the lesion had healed and showed complete resolution. The patient has been followed for 2 years and had no recurrence.

\section{Discussion}

The global prevalence of human TB due to $M$. bovis has been estimated at $3.1 \%$ of all human TB cases [2]. According to the report of the European Food Safety Authority, Greece is not officially bovine tuberculosis-free in cattle, but no cases of human tuberculosis were confirmed, to our knowledge, during the last 10 years [6]. As stated by the above report, several human cases of bovine TB have been reported from other European countries which have been approved as being free from bovine $T B$ in cattle like Germany or Austria [6]. It is possible that human bovine TB is under-diagnosed in our settings due to difficulties in obtaining samples or differentiating the organism from $M$. tuberculosis in culture.

People can be infected with $M$. bovis, by inhalation of tubercle bacilli or by consumption of infected products. 
Pulmonary tuberculosis due to $M$. bovis is more common in rural dwellers or people with occupational exposure, as a result of inhalation of dust particles or bacteria-containing aerosols shed by infected animals [2]. Those who live in urban areas usually acquire the infection via the gastrointestinal route due to consumption of poorly cooked meat, unpasteurized milk, or milk products and develop extrapulmonary tuberculosis [2, 3]. Although, human-to-human transmission of $M$. bovis has been reported $[2,4]$, this mode of transmission is rare and is likely to occur in populations particularly susceptible to tuberculosis such as the HIV-infected people [4].

Tuberculosis of the oral cavity is extremely rare and thus presents a diagnostic challenge. The intact oral mucous membrane presents a natural resistance barrier to mycobacterial invasion, and any break or loss of this barrier may provide a route of entry [5]. It is most likely that, in the case presented, the patient had primary oral tuberculosis since there was no pulmonary involvement. It is assumed that the mycobacterium was directly inoculated into the dental socket and the mandible through the decayed primary tooth, causing infection and abscess formation with subsequent spread to the submandibular space [5]. Following surgical treatment, there was some improvement initially but since the cause of the mandibular infection was not treated, symptoms persisted, and soon, mandibular osteomyelitis was obvious radiographically.

The isolation of $M$. bovis from an immunocompetent child residing in an urban area raises the question of how the infection was acquired. There was no history of unpasteurized dairy products consumption, but this possibility cannot be ruled out since most cases with primary oral tuberculosis in the literature were due to consumption of infected milk or milk products [2]. By analogy, history of consumption of unpasteurized dairy products is negative in about $20 \%$ of cases with brucellosis, another zoonosis [10]. Human-tohuman transmission from an ill person could not be excluded. However, the likelihood of reactivation of an old bovine tuberculosis infection in family members was considered unlikely after thorough investigation. Finally, transmission of M. bovis infection after dental manipulations has been reported, but our patient did not have such a history [5].

The identification of MTBC isolates depends on biochemical testing, drug susceptibility testing, and DNA techniques [1]. Resistance to PZA is a major criterion for the differentiation of $M$. bovis subsp. bovis and must raise suspicion in the appropriate epidemiological setting. The definite diagnosis and identification was facilitated in our case by the application of a molecular genetic approach which is a useful tool for the differentiation at species/strain level of the closely related members of MTBC.

Additionally, QFT-IT test was positive in this case. This test measures interferon gamma production from a person's white blood cells in response to stimulation with antigens specific to
MTBC complex organisms including $M$. bovis, but absent from all $M$. bovis Bacillus Calmette-Guérin strains and most nontuberculous Mycobacterium species [1]. This method can detect MTBC infection but can neither differentiate between species nor between infection and active disease.

In conclusion, we report a case of dental abscess due to $M$. bovis in an immunocompetent child who lacked epidemiological risk factors for this infection. The inclusion of tuberculosis in the differential diagnosis of unclear pulmonary and extrapulmonary disease remains important, and tuberculosis has to be managed according to international standards [7]. Although, childhood tuberculosis represents an important part of the disease burden, yet its diagnosis remains challenging [9]. The application of molecular genetic techniques that can easily differentiate tubercle bacilli belonging to $M T B C$ could also specify the true prevalence of $M$. bovis infections that are currently underestimated.

Acknowledgements We thank the staff of microbiology laboratory of Sotiria Chest Hospital for the isolation of the M. bovis strain. No financial support was required for the present study.

Open Access This article is distributed under the terms of the Creative Commons Attribution Noncommercial License which permits any noncommercial use, distribution, and reproduction in any medium, provided the original author(s) and source are credited.

\section{References}

1. Andersen P, Munk M, Pollock J et al (2000) Specific immunebased diagnosis of tuberculosis. Lancet 356:1099-1104

2. Ayele WY, Neill SD, Zinsstag J et al (2004) Bovine tuberculosis: an old disease but a new treat to Africa. Int J Tuberc LungDis 8:924-937

3. Baker MG, Lopez LD, Cannon MC et al (2006) Continuing Mycobacterium bovis transmission from animals to humans in New Zealand. Epidemiol Infect 134:1068-1073

4. Blázquez J, de Los E, Monteros LE, Samper S et al (1997) Genetic characterization of multidrug-resistant Mycobacterium bovis strains from a hospital outbreak involving human immunodeficiency virus-positive patients. J Clin Microbiol 35:1390-1393

5. Ebenezer J, Samuel R, Mathew GC et al (2006) Primary oral tuberculosis: report of two cases. Indian J Dent Res 1:41-44

6. EFSA (2005) The community summary reports on trends and sources of zoonose, zoonotic agents, antimicribial resistanceand foodborne outbreaks in the European Union in 2005. Available at: www.efsa.europa.eu

7. Magdorf K, Detjen AK (2008) Proposed management of childhood tuberculosis in low-incidence countries. Eur J Pediatr 167:927-938

8. Richter E, Weizenegger M, Fahr AM et al (2004) Usefulness of the geno type MBTC assay for differentiating species of the Mycobacterium tuberculosis complex in cultures obtained from clinical specimens. J Clin Microbiol 42:4303-4306

9. Rigouts L (2009) Clinical practice: diagnosis of childhood tuberculosis. Eur J Pediatr 168:1285-1290

10. Tsolia M, Drakonaki S, Messaritaki A et al (2002) Clinical features, complications and treatment outcome of childhood brucellosis in central Greece. J Infect 44:257-262 